\title{
Efecto de la danza en la mejora de la autoestima y el autoconcepto en niños, niñas y adolescentes: Una revisión Effect of dance on improving self-esteem and self-concept in children and adolescents: A review
}

*N ormaVergaraVilchez, *Alba Fuentes Sandoval, *Héctor Gonzales Chacana, **, ***Cynthia Cadagan Fuentes, **** Solange MoralesYañez, $* * * * *$ Carolina Poblete Gálvez, $*, * * * *$ Carlos Emilio PobleteAro * Universidad de las Américas(Chile), ** U niversidad Andrés Bello(Chile), ***U niversidad Gabriela

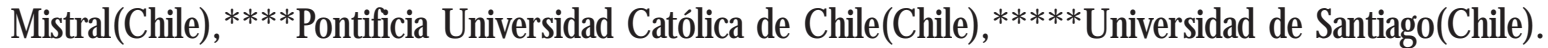

Resumen. La adolescencia es un período de transición, comprendido entre la niñez y la etapa adulta, en la cual se presentan cambiosfísicos, emocional es biopsicosociales que gener an inestabilidad eincertidumbre. En ocasiones esto puede desembocar en el desarrollo de una baja autoestima producto de un autoconcepto o autopercepción sesgada por los contextos de vida. Esto puede traer consecuencias negativas para la vida escolar ya sea por una mala convivencia con sus pares, desarrollar poca confianza para exponer ideas o sentimientos negativos hacia su propia persona, entre otros. La danza y sus efectos en la autopercepción de adolescentes será abordada en este artículo sobre la base de una investigación de revisión bibliográica, real izada en diversas bases de datos que tuvo por objetivo identificar aquellos artículos que pudiesen ayudarnos a determinar en particular el efecto de la danza en la autopercepción y la autoestima en programas de intervención, es decir, sobre la base de las experiencias, más allá de la teoría. Posterior a la búsqueda bibliográfica, sólo 5 artículos se refieren en específico al objetivo planteado, lo que refuerzala idea del rol de este tipo de prácticas en la etapa escolar y una serie de consideracionesa mencionar en esteartículo. También surgen cuestionamientos acercadelas escasas investigaciones deeste tipo en estaáreay de la importancia de incluir multifactores de análisis en el desarrollo de la autoestima y autoconcepto.

Palabras Clave: Danza, autoestima, autoconcepto, autopercepción.

Abstract. Adolescence is a transition period, between childhood and adulthood, in which physical, emotional, and biopsychosocial changes occur that generate instability and uncertainty. Sometimes this can lead to the development of low self-esteem as a result of a self-concept or self-perception biased by life contexts. This can have negative consequences for school life, either due to poor coexistence with peers, developing little confidence to expose negative ideas or feelings towards their own person among others. Dance and its effects on the self-perception of adolescents will be addressed in this article based on a bibliographic review investigation carried out in various databases that aimed to identify those articles that could help us determine in particular the effect of dance on Self-perception and self-esteem in inter vention programs, that is, on the basis of experiences beyond theory. Of the result, 5 articles refer specifically to the proposed objective, which reinforces the idea of the role of this type of practice in the school stage and a series of considerations to be mentioned in this article. Q uestions al so arise about the scarcity of research of this type in this area and the importance of including multifactor analysis in the development of self-esteem and self-concept.

Key Words: Dancing, self-conception, self-perception.

\section{Introducción}

La adolescencia es uno de los periodos con mayores cambios en el ser humano desde el punto de vista biopsicosocial. Cambios hormonales y morfo estructurales condicionan a la persona a desarrollar nuevas rela ciones sociales que ponen en tensión su identidad, la aceptación propia y la de los otros (Iglesias, 2013). Es el periodo en el que la búsqueda de identidad puede con-

Fecha recepción: 31-05-20. Fecha de aceptación: 16-11-20

Carlos Emilio Poblete Aro

carlos.poblete.aro@gmail.com dicionar la autoestima, pues los adolescentes se enfrentan a un periodo de adaptaciones y de búsqueda y entendimiento de sí mismos(as). La familia y el entorno de desarrollo como la escuela son fundamentales en la consolidación de una identidad y autoestima positiva. En estudios de Centroamérica se pone de manifiesto que los contextos familiares son determinantes en estos niveles de autoestima (Álvarez, Herrera, Herrera, Villalobos \& Araya, 2019) llegando a ser, para esta realidad local, un problema de salud mental dada las condiciones de privación social en la que se encuentran, lo que además conlleva a ciertos trastornos de la imagen corporal. También lo corroboran Sánchez \& Ruiz (2015) 
y Taberno, Serrano y M érida (2017), al señalar que los ambientes de inseguridad social, como puede ser la fa milias y amistades cercanas, influencian tempranamente en la construcción de una salud mental apropiada. A luz de esta importante llamada de alerta acerca de la influencia que pueden tener niños, niñas y adolescentes en sus entornos cercanos de desarrollo es que surgen dos elementos como determinantes para ser analizado en el contexto de la danza y sus posibilidades en el aula: la autoestima y el autoconcepto.

\section{Antecedentes}

La autoestima corresponde a la «valoración individual que cada persona posee de sí mismo, que se ve influenciada por el conjunto de estados anímicos presentes en un relativo periodo de tiempo» (SilvaEscorcia, 2015). Así también, Cubas-Martinez, MarcoAhulló, M onfort-Torres, Villarrasa-Sapiña, Pardo-Ibañez y García-Massó (2019) resaltan que es el factor más influyente en las relaciones sociales sobre la base de un estudio en niños, niñas y preadolescentes. El autoconcepto, por su parte, abarca tres grandes áreas, la cognitiva que hace referencia a las múltiples identidades 0 ideas que lo forman, el área afectiva que construye la autoestima y la tercera, el área conductual que es la autoafirmación o búsqueda del reconocimiento propio o de los demás (Navarro, Tomás \& O liver, 2006; Navarro, 2009).

Existen factores que influyen en el autoconcepto y la autoestima. Estos son en gran medida producto de las experiencias previas en la etapa de la infancia tanto de quienes rodean a los niños, niñas y adolescentes, ya sea familiares u otras personas (Tabernero, Serrano, \& Mérida, 2017) o de su entorno en general. El primero de ellos, la familia, influye fuertemente en la forma ción de la autoestima adolescente (M ontes, Vaca \& Rubio, 2007). Este proceso depende mucho del clima fa miliar y del tipo de comunicación efectiva que se desa rrolle al interior del núcleo. Esto incluye el apoyo familiar que le permita al adolescente creer en sí mismo (a), el contexto económico y el capital cultural en el que se desenvuelva la familia (N avarro, 2009).

Por otra parte, los jóvenes con baja autoestima se caracterizan también por presentar una disminuida salud física (Rodríguez \& Caño, 2012) lo que puede condicionar también su autoconcepto y autoestima. En un estudio realizado en Chile, en estudiantes de $70^{\circ}$ a $4^{\circ}$ medio, reveló que más del $43 \%$ considera que tiene mala suerte, cerca de un $76 \%$ no cree tener liderazgo y un $61 \%$ no se siente popular entre sus pares. Estas cifras dan cuenta de que la juventud en Chile posee una baja autoestima, factor importante para el desenvolvimiento de estos jóvenesen lavida (Gorostegui \& Dörr, 2009). Estos resultados son alarmantes, dado que es posible identificar en la literatura que una alta autoestima y autoconfianza disminuye los síntomas psicopatológicos asociados a la ansiedad, síntomas depresivos y desesperación al momento de enfrentar un desafío, lo que permite una gran expectativa de su futuro. Además, permite disfrutar de experiencias positivas y que son más eficaces al momento de confrontar experiencias que puedan ser negativas (Rodríguez \& Caño, 2012). Es por tanto un tema que requiere de una revisión y un análisis a considerar.

Contextualmente se podría decir que si un adolescente es apoyado emocionalmente por su familia, compañeros y compañeras, tendrá un aumento en su autoestima y su potencial para sortear las dificultades será mejor. Este potencial se verá reflejado en la especificidad que logre tener de su autoconcepto, es decir, el conocimiento de sí mismo, desde un mirada física, social y psicológica. Shavelson, Hubner y Stanton (1976) señalan que el autoconcepto es una autoconcepción jerárquica y multidimensional que se divide según Fox y Corbin (1989) e Infante y Goñi (2009) en cuatro dimensiones: lahabilidad física, lacondición física, el atractivo físico y la fuerza. Estas dimensiones requieren ser analizadas como lo sugiere Revueltas, Esnaola y Goñi (2016) en relación con otras variables como la actividad física. Es por lo anterior que en la presente revisión bibliográfica, se centra en un aspecto bastante particular de lo que podría denominarse actividad física, pues la Danza es una manifestación de la motricidad humana que comprende tanto la realización de actividad física además de ser una herramienta para el desarrollo biopsicosocial. En este trabajo, abordaremos la danza desde un punto de vista biopsicosocial y mostraremos la evidencia relacionada con estos dos conceptos, la autoestima y el autoconcepto en la práctica de la danza y el contexto escolar, y cómo puede influir en estos dos factores.

\section{Estado actual del tema}

En diversas ocasiones, se pueden presentar en el ámbito escolar distintas experiencias que pueden generar en losestudiantesel desarrollo de unabajaautoestima, por ejemplo: una mala convivencia en el aula, el desinterés en todo tipo de práctica y propuesta lúdica-depor- 
tiva, la poca confianza para exponer ideas y sentimientos en actividades que involucren la comunicación no verbal, por no considerase lo suficientemente competentes o seguros para tomar decisiones. Este tipo de situaciones que se dan en la escuela trae como consecuencia un bajo rendimiento académico ( $M$ ontes, Vaca, $\&$ Rubio ,2007) pues la mirada de la o el docente muchas veces está sobre la base del comportamiento esperado, más que la mirada hacia la autoestima y/ 0 autoconcepto que poseen sus estudiantes como factor explicativo de sus acciones. Sin embargo, por otro lado, un buen autoconcepto y los logros académicos se refuerzan mutuamente, y cada uno de ellos conduce a ga nancias en el otro. Marsh \& Craven (2016) señalan que la danza es una herramienta en la educación física menos convencional, pero que puede impulsar la consideración y profundización defactores como el autoconcepto, el aprendizaje lo que puede aumentar los logros académicos. Por otra parte, se considera que la actividad física se relaciona con los niveles de autoestima (Álvarez, et. al., 2019) aunque en términos moderados como advierte Cascales y Prieto (2018), lo cierto es que no toda actividad física es pertinente para estimular el autoconcepto positivo y la autoestima, pues habría que poner bastante atención a los niveles de competitividad que pudiesen generar mayor violencia y con ellos mermar esfuerzos demejorar laautoestima (C Cubas-M artinez et. al., 2019).

Es por lo anterior que es relevante buscar herramientas metodológicas que permitan mejorar el autoconcepto y la autoestima del estudiante en el aula escolar, ya que permiten tomar mejores decisiones, sa ber aprovechar las oportunidades que se les presentan y construir un cimiento sólido para entrar ala vidaadulta de forma satisfactoria y exitosa (Navarro, 2009). Una de las maneras de fortalecer la autoestima en el ámbito escolar es ofrecer espacios y experiencias en las cuales el estudiante pueda desarrollarse integralmente. En este contexto, la danza se presenta como una oportunidad de desarrollo tanto integral y desde lo personal, ya que permite una adecuada interacción entre pares, lo que ayuda al bienestar de los adolescentes (Vicente, U reña, Gómez \& Carrillo, 2010; Sánchez \& Lluch, 2011).

La autoestima en los niños, las niñas y los adolescentes se debe considerar como un desafío a desarrollar en toda propuesta metodológica de enseñanza-aprendiza je, por ello concentramos la mirada en la danza, pues es, en su acción, un camino de construcción de la ima gen corporal de quien lo practica, pues son las sensaciones y relaciones con el entorno las que se demandan principalmente (Requena-Pérez, M artín-Cuadrado, \& Lago-Marín, 2015).

La danza es una herramienta para aumentar la autoestima. Ella ayuda a desarrollar tres grandes áreas: la social, pues permite generar lazos y relaciones interpersonales; la motriz, contribuye a las tareas motrices cotidianas y la cognitiva, aporta herramientas que estimulan la actividad mental en los movimientos ejecutados corporalmente (Vicente et al., 2010). Permite conectar cuerpo, mente y alma y traspasar este bienestar hacia los demás. La experiencia de aprender a bailar proporciona sentimientos positivos que permiten aumentar la autoconfianza. Cuando se aprende un nuevo paso, un movimiento o una nueva rutina de baile, el adolescente se da cuenta que es capaz de aprender cosas nuevasy superar nuevos desafíosy obstáculos (Fructuoso \& Gómez, 2001). El nivel de seguridad aumenta con estas experiencias de aprendizaje y eventualmente esa seguridad, se refleja en la autoestima y en general, en la vida real.

Es fundamental que los adolescentes participen en la mayor cantidad de actividades posibles, que le permitan un adecuado desarrollo motor, autoestima y autoconcepto en el ámbito educativo, siendo la danza una herramienta viable, considerando que hay estudios que afirman que una intervención de danza, mejora significativamente la percepción y comprensión de las emociones y del propio cuerpo (Rueda\& López, 2013). La danza enriquece modos y medios de comunicación, estructura de la personalidad, desarrollo social, crea lazos entre los compañeros, fortalece relaciones humanas, evidenciando mejoras en sus capacidades motoras y creativas ayudando a expresar su verdadera identidad (Rueda \& López, 2013; Requena-Pérez et al., 2015; Sánchez et al., 2011).

Se puede entender desde la teoría que la danza es un elemento valioso para todo proceso de formación del ser humano, destinado a conseguir personas seguras, con posibilidades de actuar en el mundo y responsables en su relación con los demás (Fructuoso \& Gómez, 2001). En este sentido nos preguntamos por la evidencia científica de programas de intervención específica para la danza y su relación con la autoestima y autoconcepto. Es necesario señalar que tanto los contextos como las diversidades presentes en el aula, requieren de la mirada crítica de las posibilidades que nos pueda ofrecer este tipo de experiencias pedagógicas, sin embargo creemos que el camino llevado a cabo en diversas realidades, nos pueden aportar ciertas consideraciones ineludibles que sobre la base de la sistematización e investigación rigu- 
rosa podemos avalar.

Por lo tanto, el objetivo de esta revisión fue identificar a través de una búsqueda bibliográficas, programas de intervención y/ 0 experiencias pedagógicas que nos permitan mirar desde lo experiencial, el efecto de la danza en el autoconcepto y autoestima en niños, niñas y adolescentes, que nos orienten en el ejercicio de este tipo de propuesta y aplicarla sobre la base de la experiencia que nos permita respetar el contexto escolar.

La búsqueda se realizó en las bases de datos PubM ed, Scopus, Wos y Scielo con las palabras claves «Dancing» AND ( «Adolescent»OR «Child») AND «self conception» $O R$ «self perception» $O R$ « self esteem» $O R$ «self confidence» $O R$ «self efficacy», considerando artículos en inglés y en español. Adicionalmente se rea lizó una búsqueda manual revisando las referencias de los artículos encontrados, posteriormente se realizó una búsqueda informal. La búsqueda de datos consistió en establecer artículos publicados con un rango de 10 años. La búsqueda se realizó con fecha 20 de septiembre del 2020. Al finalizar la búsqueda se identificaron 173 registros que concuerdan con las palabras claves menciona das anteriormente.

Para la búsqueda se incluyeron artículos desde el año 2009 hasta el 2020. Como criterios de inclusión, se incluyeron trabajos de programas de intervención para mejoras de la autoestima y/ 0 autoconcepto a través de la danza en niños, niñas y adolescentes de entre los once a los 18 años. En cuanto al tipo de estudio se consideraron los clínicos aleatorizados y cuasi-experimentales, en cuanto al tipo de intervención que fueran talleres dirigidos, que proporcionarán todo tipo de resultados (positivos y negativos) y en idioma inglés o español. Para considerar una muestra homogénea, similar a lo que se observa en una escuela, se consideraron como criterios de exclusión, personas mayores de 18 años y menores de 11 años con al guna discapacidad motora, además de trabajos que havan incluido a estudiantes deportistas, bailarines de élite 0 activida des del tipo fitness dirigido como «Baile entretenido» o Zumba. Además, se excluyeron estudios cuasiexperimentales, capítulos de libros, revisiones sistemáticas, meta análisis y casos clínicos.

La forma de identificación bibliográ fica procede de la siguiente forma: en la etapa 1, de un total de 173 registros encontrados en las bases de datos, se resta ron por medio de EndNote, 23 archivos duplicados quedando 150 archivos. En la etapa 2 se revisaron los títulos y los abstract de los 150 archivos seleccionados, de los cual es 49 cumplían con nuestro outcome. En la etapa3, de los 49 archivos, posterior a cada lectura se descartaron 44 archivos por no cumplir con nuestros criterios de inclusión y exclusión; 7 por revisiones bibliográficas, 1 por ser caso clínico, 1 por meta-análisis, 4 por ser estudios cualitativos, 15 por tener en cuenta deportistas y bailarines de élite, 10 por rango de edad, 1 por idioma y 5 por medir un outcome diferente, por lo que fueron incluidos en esta revisión 5 artículos.

\section{Resultados y conclusiones}

De un total de 173 registros. Sólo 5 artículos cumplieron con los criterios de inclusión y exclusión, de los cuales 5 demuestran que la danza mejora la autoestima, 1 demuestra la mejora en las relaciones interpersonales, 1 demuestra la mejora en los niveles de satisfacción y de autoconfianza, 1 disminuye los niveles de ansiedad somática y cognitiva, 1 mejora el clima motivador.

1) En el estudio de Rokka, Kouli, Bebetsos, Goulimaris y Mavridis (2019) un total de 160 alumnos entre doce y 13 años de edad fue dividido en dos grupos, uno experimental que constaba de 91 alumnos y otro grupo control que constaba de 69 al umnos. El programa de intervención tuvo un total de 10 semanas en las cua les se impartieron 3 clases por semana con una dura ción de 45 minutos cada una. Se realizó un cuestionario para medir la motivación intrínseca. Además, se hizo una evaluación inicial y una final para poder medir y analizar la motivación intrínseca y el clima motivador. Para ello se utilizó la siguiente herramienta IMI (Inventario de Motivación Intrínseca, M cauley, Duncan \& Tammen, 1989). Posterior a la intervención, se observó una mejora en el clima motivador en las clases de educación física. Además, se observó una correlación positiva de este resultado con la motivación intrínseca.

\begin{tabular}{|c|c|c|c|c|c|}
\hline Autores Características en base a la escala de PED ro & $\begin{array}{l}\text { (Wagener Et } \\
\text { Al., 2012) }\end{array}$ & $\begin{array}{l}\text { (Rokka EtAl., } \\
\text { 2019) }\end{array}$ & $\begin{array}{l}\text { (Filippou et } \\
\text { al., 2018) }\end{array}$ & $\begin{array}{l}\text { (Jago et al., } \\
\text { 2015) }\end{array}$ & $\begin{array}{l}\text { (Duberg et } \\
\text { al., 2013) }\end{array}$ \\
\hline 1. Criterios de elección fueron especificados & $\mathrm{SI}$ & SI & SI & $\mathrm{SI}$ & SI \\
\hline 2. Los sujetos fueron asignados al azar a los grupos & SI & $\mathrm{SI}$ & SI & SI & SI \\
\hline 3. La asignación fue oculta & NO & NO & NO & NO & SI \\
\hline 4. Grupos fueron similares al inicio de la intervención & SI & SI & NO & SI & SI \\
\hline 5. Los sujetos fueron cegados & NO & NO & NO & NO & NO \\
\hline 6. Los terapeutas fueron cegados & SI & NO & NO & NO & NO \\
\hline $\begin{array}{l}\text { 7. Los evaluadores que eval uaron al menos un } \\
\text { resultado clave fueron cegados }\end{array}$ & NO & NO & NO & Sl & NO \\
\hline $\begin{array}{l}\text { 8. Estudio terminó con más del } 85 \% \text { de la muestra } \\
\text { inicial }\end{array}$ & $\mathrm{SI}$ & $\begin{array}{c}\text { NO } \\
\text { No lo declara }\end{array}$ & $\begin{array}{c}\text { No } \\
\text { No lo declara }\end{array}$ & SI & NO \\
\hline $\begin{array}{l}\text { 9. Presentaron todos los datos de sujetos que } \\
\text { participaron o se real izó el anál isis de intención a tratar }\end{array}$ & $\begin{array}{l}\text { NO } \\
\text { r No lo declara }\end{array}$ & SI & SI & $\begin{array}{l}\text { NO } \\
\text { No lo declara }\end{array}$ & $\begin{array}{l}\text { NO } \\
\text { No lo declara }\end{array}$ \\
\hline $\begin{array}{l}\text { 10. Resultados de comparación estadísticas en al } \\
\text { menos un resultados clave }\end{array}$ & $\mathrm{Sl}$ & SI & SI & SI & SI \\
\hline $\begin{array}{l}\text { 11. Estudio presenta medidas puntuales y la } \\
\text { variabilidad de al menos un resultado clave }\end{array}$ & $\mathrm{SI}$ & SI & SI & SI & SI \\
\hline Puntos totales & $7 / 11$ & $6 / 11$ & $5 / 11$ & $7 / 11$ & $7 / 11$ \\
\hline
\end{tabular}


2) En el Artículo Filippou, Rokka, Pitsi, Gargalianos, Bebetsos y Filippou (2018) un total de 260 estudiantes de $8 v 0$ grado de educación media fueron dividido en dos grupos; uno experimental que constaba de 76 alumnos y 68 alumnas y otro grupo control compuesto por 58 alumnos y 58 alumnas. La intervención del progra ma duró 4 semanas con 2 sesiones cada una, con una duración de 45 minutos por clases. Se analizó que en ambos grupos los niveles de satisfacción y autoconfianza mejoraron significativamente mientras que los niveles de ansiedad somática y cognitiva disminuyeron. Para poder concluir con dicha información se utilizó el cuestionario de inventario de ansiedad 2 de Duda \& Nicholls(1992).

3) En el estudio de Duberg, Hagberg, Sunvisson, y Möller (2013), se realizó una investigación en la que participaron niñas de 13 a 18 años con problemas de internalización como cuadro de estrés y síntomas psicosomáticos. Se asignaron dos tipos de grupo, uno de intervención que fue un total de 59 chicas y el otro fue el grupo control que se conformó por 53 niñas. La intervención que se produjo en este estudio consistió en clases de bailes realizadas dos veces por semana en un periodo de 8 meses. Cada sesión duró 75 minutos. Se demostró que la salud autoevaluada mejora en adolescentes con problemas de internalización e incluso esta mejora se mantiene un año después de la inter vención. Para este resultado se utilizaron cuestionarios de línea base, un cuestionario de 88 preguntas sobre el estilo de vida y un cuestionario no declarado.

4) En el trabajo de Jago, Edwards, Sebire,Tomkinson, Bird, Banfield y Blair (2015) participó una población de 571 niñas entre 11 y 12 años de 18 escuelas secundarias. Se realizaron 40 sesiones de danza extraescolar de 75 minutos cada una, durante un total de 20 semanas. Se dividieron en dos grupos, un grupo control y un grupo de intervención. Se observó un aumento de la autoestima en el grupo intervenido en comparación al grupo control. Para la evaluación de calidad de vida se utilizó un formulario EQ-5D-Y.

5) En lainvestigación deWagener, Fedele, Mignogna, Hester y Gillaspy (2012), participaron 40 adolescentes entre 12 y 18 años en sesiones de Exergaming durante un periodo de 10 semanas. Se realizaron 3 sesiones por semanas con una duración de 40 minutos cada una. Se crearon dos grupos de 20 adolescentes cada uno, el primer grupo fue control aleatorizado, y el otro grupo estaba compuesto por adolescentes que frecuentemente practicaban Exergaming. Se observó una mejora en la autoestima y en las relaciones interpersonales de los adolescentes utilizando la Evaluación del Instrumento Behavior Assessment System for Children, Second Edition (BASC-2).

De la revisión de estas investigaciones se desprende principalmente que estos estudios a través de su intervención lograron evaluar el efecto de la danza en la autoestima en niños y adolescentes entre 11 y 18 años. Se excluyeron deportistas y bailarines de élite. Al rea lizar una mirada comparativa de los aportes de dichos estudios es posible identificar evidentes niveles de mejoras en la autoestima y autoimagen 0 autoconcepto del estudiantado que participa de actividades de danza, así como un aumento en los niveles y las posibilidades de socialización.

\section{Aplicaciones prácticas en base a la revisión}

Los principales resultados indican que los programas de intervención de danza:

- Aumentaron los niveles de autoestima (Jago et al., 2015; Wagener et al., 2012).

- Disminuye la ansiedad (Filippou et al., 2018).

- Aumenta la satisfacción en los jóvenes (Filippou et al., 2018) y,

Tabla 2 .

Resumen de los artículos seleccionados

\begin{tabular}{|c|c|c|c|}
\hline Autor & Población & Intervención & Resultados Principales \\
\hline $\begin{array}{l}\text { (Rokka et al., } \\
\text { 2019) }\end{array}$ & $\begin{array}{l}160 \text { Alumnos de } 12 \text { y } 13 \text { años. } \\
\text { Grupo experimental: } 91 \text { alumnos; ( } 46 \text { niños; } 45 \text { niñas). } \\
\text { Grupo Control: } 69 \text { alumnos ( } 32 \text { niños y } 37 \text { niñas) }\end{array}$ & $\begin{array}{l}\text { El programa de inter vención duró } 10 \\
\text { semanas, de las cuales por semanas se } \\
\text { realizaban } 3 \text { clases de } 45 \text { minutos. }\end{array}$ & $\begin{array}{l}\text { } \uparrow \text { El clima motivador en las clases de educación física tuvo una cor relación positiva } \\
\text { significativa con la motivación intrínseca. } \\
\text { IM I (Inventario de M otivación Intrínseca, M cAuley, D uncan y Tammen, } 1989\end{array}$ \\
\hline $\begin{array}{l}\text { (Filippou et al., } \\
\text { 2018) }\end{array}$ & $\begin{array}{l}\text {, } 260 \text { estudiantes de } 8 v 0 \text { grado de educación media divididos } \\
\text { en Grupo experimental: compuesto por } 76 \text { al umnos y } 68 \\
\text { mujeres. Grupo control: } 58 \text { hombres y } 58 \text { mujeres }\end{array}$ & $\begin{array}{l}\text { El Programa de intervención duró: } 4 \\
\text { semanas; } 2 \text { clases por semana de } 45 \\
\text { minutos cada clase. }\end{array}$ & $\begin{array}{l}\uparrow \text { Niveles de satisfacción y autoconfianza. } \\
\downarrow \text { Niveles de ansiedad somática y cognitiva. Se utilizaron el cuestionario de satisfacción de } \\
\text { Inventario de ansiedad } \mathbf{2} \text { y Duda y Nicholls }\end{array}$ \\
\hline $\begin{array}{l}\text { (Jago et al., } \\
2015)\end{array}$ & $\begin{array}{l}571 \text { Niñas entre } 11 \text { y } 12 \text { años de } 18 \text { escuelas secundarias } \\
\text { aceptaron participar de la investigación. }\end{array}$ & $\begin{array}{l}40 \text { sesiones de danza de } 75 \text { minutos cada } \\
\text { una, durante } 20 \text { semanas. }\end{array}$ & $\begin{array}{l}\uparrow \text { El grupo de intervención tiene un aumento en la autoestima en comparación al grupo } \\
\text { control } \\
\text { Formulario EQ-5D-Y para la evaluación de calidad de vida }\end{array}$ \\
\hline $\begin{array}{l}\text { (Duberg et al., } \\
\text { 2013) }\end{array}$ & $\begin{array}{l}\text { Adolescentes mujeres entre } 13 \text { y } 18 \text { años divididos en : } \\
-59 \text { niñas para la intervención } \\
-53 \text { niñas como grupo control }\end{array}$ & $\begin{array}{l}8 \text { meses, durante dos veces por semana, } \\
\text { cada clase duró } 75 \text { minutos }\end{array}$ & $\begin{array}{l}\uparrow \text { Mejora la salud autoevaluada de las adolescentes con problemas de internal ización, la } \\
\text { mejora se mantuvo un año después de la intervención. } \\
\text { Cuestionarios de línea base, cuestionario de } 88 \text { preguntas sobre el estilo de } \\
\text { vida, cuestionario no declarado } \\
\text { La salud autoeval uada se midió con el ítem individual pregunta ¿Cómo } \\
\text { califica su salud general? }\end{array}$ \\
\hline $\begin{array}{l}\text { (Wagener et } \\
\text { al., 2012) }\end{array}$ & $\begin{array}{l}\text { Se eval uaron } 40 \text { adolescentes obesos entre } 12-18 \text { años. } \\
\text { Aleatorizados } 20 \text { exergaming y } 20 \text { grupo de control. }\end{array}$ & $\begin{array}{l}10 \text { semanas de exergam } \epsilon . \\
3 \text { veces por semana sesiones de } 40 \\
\text { minutos. }\end{array}$ & $\begin{array}{l}\uparrow \text { Laautoestima. } \\
\uparrow \text { Relaciones inter per sonales. } \\
\text { Evaluación del Instrumento Behavior Assessment System for Children, } \\
\text { Second Edition (BASC-2) }\end{array}$ \\
\hline
\end{tabular}


- M ejora el clima motivacional junto con las rela ciones interpersonales (Rokka et al., 2019; Wagener et al. , 2012)

Es importante destacar que en la comparación rea lizada entre las intervenciones de danza con las clases de educación física, los niños, las niñasy los adolescentes mencionan que la danza no tiene un carácter competitivo, lo que la hace estar más orientada a la participación libre y espontánea, lo que produce una sensación de pla cer y libertad en los estudiantes (Rokka et al., 2019). Además, diversas investigaciones, refuerzan que el factor «esfuerzo físico», incrementa cuando la clase se torna interesante y placentera, por lo tanto, las investiga ciones proponen que dentro de las clases de educación física es importante generar la motivación en los estudiantes para obtener un clima confiable, y evaluar las respuestas positivas al esfuerzo físico. En efecto, la danza puede ofrecer múltiples beneficios. Rokka et. al. (2019), utilizó el Inventario de Motivación Intrínseca (IMI) en los adolescentes para medir el clima motivador en las clases de educación física y observó una mejora en el clima motivacional. También, observó la satisfacción de los estudiantes que participaron en las clases de danza, los cuales redujeron sus niveles de ansiedad y aumentaron la confianza en sí mismos, lo que ayudó a potenciar la autoestima de los estudiantes y los ayudó a relacionarse demaneraarmónica con suspares(Filippou et al., 2018).

La danza supone una oportunidad para generar relaciones interpersonales. Esto permite a quienes la practican crear nuevos lazos de amistad, pues se utiliza el tiempo libre con personas que tienen intereses y características similares. De esta forma, se potencia la autoconfianza, junto con disminuir los niveles de ansiedad e incrementar la satisfacción y autoestima de los niños, las niñas y los adolescentes. Además, mejora el rendimiento académico y se pueden prevenir disfunciones sociales, el consumo de sustancias ilícitas e incluso suicidios (Fructuoso \& Gómez, 2001; Sánchez et al., 2011) Estos resultados nos impulsan a reafirmar el carácter multifactorial de este tipo de prácticas, las que actúan favorablemente en diversos aspectos de la salud de las per sonas, no solo los considerados en este artícu10.

En este sentido, los resultados observados en los artículos analizados indican que la danza puede mejorar o proporcionar aspectos innovadores a la clase de educación física, lo cual sugiere que las escuelas puedan considerar los programas de danzas para poder optimizar no solo la satisfacción y autoestima de los niños, las niñas y los adolescentes en base a las posibilidades de explora ción y comprensión de la danza, sino que además mejorar considerablemente la memoria motriz y la expresión corporal de ellos y ellas. Las investigaciones analizadas declaran los beneficios que la danza proporciona a las y losjóvenestanto a nivel social como personal, pues esta permite que aumenten su autoestima, la autoimagen y el autoconcepto que ellostienen de sí mismos (Duberg et al., 2013; Filippou et al., 2018; Jago et al., 2015; Rokka et al., 2019; Wagener et al., 2012).

Si bien los resultados en esta revisión muestran mejoras en la autoestima y el clima motivacional de los estudiantes, los trabajos incluidos en esta revisión presentan metodologías heterogéneas, por lo que es difícil plantear una propuesta de intervención para los niños, las niñas y los adolescentes con parámetros únicos. Sin embargo, debido a que los trabajos mostraron mejoras significativas en los resultados asociados a la autoestima durante la realización de la danza entre cuatro a 10 semanas, se sugiere que los programas de danza con el objetivo de mejorar la autoestima deben tener, al menos, una duración de 4 semanas, 2 sesiones por semana con una duración de 45 minutos, para poder visualizar cambios y mejoras significativas en la autoestima de los niños, las niñas y los adolescentes, independientemente del modelo de danza seleccionado.

Uno de los aspectos a destacar es la ausencia de evidencia en la mejora de la composición corporal del estudiantado. Según Wagener et.al (2012) a pesar de que se ha demostrado que los y las estudiantes se sienten incentivados a participar en las clases de danza, los estudios no evidenciaron mejoras significativas en la composición corporal. Esto porqueno contemplaron un programa de alimentación paralelo al programa de danza que sirviera para reducir la ingesta calórica.

\section{Futuras líneas de investigación}

Seconcluye queladanzaes unaherramientaconfiable y viable para desarrollar y potenciar la autoestima, la seguridad en sí mismo y mejorar las relaciones huma nasentre losniños, lasniñasy los adolescentes enmarcada en el contexto escolar.

Si bien la investigación estuvo orientada hacia el desarrollo de la autoestima y la imagen corporal positiva en los niños, las niñas y los adolescentes, se debe considerar que la danza constituye una herramienta que podría mejorar componentes multifactoriales que la componen como, por ejemplo, niveles de actividad física, la composición corporal, los hábitos de alimenta- 
ción, la expresión y la comunicación. U na clase de danza constituye un enfoque de enseñanza confiable, que permite a los estudiantes tener nuevas experiencias que se orientan a la satisfacción del proceso de enseñanza y aprendizaje y además les permite aumentar la confianza en sí mismos. Esto es determinante a la hora de proyectar una imagen corporal positiva socialmente. Por tanto, sería interesante indagar en dichos multifactores a través de programas de intervención y ofrecer este tipo de actividades para enfrentar problemáticas de sa lud actual como la obesidad y el sedentarismo. Por otro lado, se podría indagar sobre la incorporación de las tecnologías, por ejemplo, videojuegos de baile, como alternativas motivacionales que encaminan a estos programas de intervención al interés de niños, niñas y adolescentes cada vez más cercanos a las tecnologías.

\section{Referencias}

Álvarez, C., Herrera Monge, M., Herrera González, E., VillalobosVíquez, G., \& ArayaVargas, G. (2019). Sobrepeso, obesidad, niveles de actividad física y autoestima de la niñez centroamericana: un análisis comparativo entre países ( 0 verweight, obesity, physical activity levels, and self-esteem in Central American children: comparative analysis between cou. Retos, 37 (37), 238-246. https:/ / doi.org/ 10.47197/ retos. v37i37.71680

Cascales, J. Á. M., \& Prieto, M. J. R. (2018). Incidencia de la práctica de actividad física y deportiva como reguladora de la violencia escolar (Incidence of the practice of physical and sporting activities as a regulator of school violence). Retos,0(35), 54-60.

Cubas-M artinez,V., M arco-Ahulló, A., M onfort-Torres, G., Villarrasa-Sapiña, I., Pardo-Ibañez, A ., \& Garcia Masso, X. (2019). Perfiles de actividad física, obesidad, autoestima y relaciones sociales del alumnado de primaria: un estudio piloto con Self-O rganizing Maps (Physical activity, obesity, self-esteem, and social relationship profiles of primary school students: a pilot s. Retos, 36(36), 146-151. https:/ / doi.org/ 10.47197/ retos. v36i36. 67549

Duberg, A., Hagberg, L., Sunvisson, H., \& Möller, M. (2013). Influencing self-rated health among adolescent girls with dance intervention: $A$ randomized controlled trial. Archives of Pediatrics and Adolescent M edicine, 167(1), 27-31. https:/ / doi. org/ 10.1001/ jamapediatrics. 2013.421

Duda, J. L., \& Nicholls, J. G. (1992). Dimensions of achievement motivation in schoolwork and sport.
Journal of Educational Psychology, 84(3), 290-299. https:/ / doi.org/ 10.1037/ / 0022-0663.84.3.290.

Filippou, F., Rokka, S., Pitsi, A., Gargalianos, D., Bebetsos, E., \& Filippou, D. A. (2018). Interdisciplinary Greek traditional dance course: Impact on student satisfaction and anxiety. International Journal of Instruction, 11(3), 363-374. https:/ / doi.org/ 10.12973/ iji.2018.11325a

Fructuoso, C., \& Gómez, C. (2001). La danza como elemento educativo en el adolescente. Apuntes Educación Física y Deportes, 66, 31-37.

Fox, K. R. , And Corbin, C. B. (1989). The Physical SelfPerception Profile: Development and preliminary validation. Journal of Sports and Exercise Psychology, 11, 408-430. DO I: 10.1123/ jsep. 11.4.408

Infante, G., \& Goñi, E. (2009). Actividad físico-deportiva y autoconcepto físico en la edad adulta. Revista de Psicodidáctica, 14(1), 49-62.

Gorostegui, M. \& Dörr, A. (2009). El peso de los factores cultural es en los resultados de la aplicación de un test norteamericano de evaluación de autoestima a un grupo de adolescentes chilenos. Castalia - Revista de Psicología de La Academia de Humanismo Cristiano, $16,65-82$.

Gregorio , N., U reña, N., Gómez, M., \& Carrillo, J. (2010). La danza en el ámbito de educativo dance in the educational context. Retos: Nuevas Tendencias En Educacion Física, Deporte y Recreacion, 17, 42-45. Retrieved from https: / / www. redalyc.org/ exportarcita. $0 a ? i d=345732283009$

Iglesias Diz, J. (2013). Desarrollo del adolescente: Aspectos físicos, psicológicos y sociales. Pediatria Integral, 17(2), 88-93.

Jago, R., Edwards, M. J., Sebire, S. J., Tomkinson, K., Bird, E. L., Banfield, K., Blair, P. S. (2015). Effect and cost of an after-school dance programme on the physical activity of 11-12 year old girls: The Bristol Girls Dance Project, a school-based cluster randomised controlled trial. International Journal of Behavioral Nutrition and Physical Activity, 12(1), 1-15. https:/ / doi.org/ 10.1186/ s12966-015-0289-y

M arsh, H.W. \& \& Craven, R. G. (2016). Reciprocal Effects of Self-Concept and Performance aM ultidimensional Perspective. Perspectives on Phychological Science, 1(2), 133-163.

M orton, N. (2009). The PED ro scale is a valid measure of the methodological quality of clinical trials: a demographic study. Australian Journal of Physiotherapy, 55(2), 129-133. https: / / doi.org/ 10.1016/ S00049514(09)70043-1 
Montes, K., Vaca, J., \& Rubio, L. (2007). Programa de Intervención para fortalecer la autoestima en adolescentes de 3er grado de secundaria. Universidad Pedagógica Nacional.

Navarro Pardo, E., Tomás Miguel, J., \& O liver Germes, A. (2006). Factores personales, familiares y académicos en niños y adolescentes con baja autoestima. Boletín de Psicología, (88), 7-26.

Navarro, M. (2009). Autoconocimiento y autoestima. Temas Para LaEducación Revista Digitales Para Profesionales de LaEnseñanza, 5(1990), 1-9. Retrieved from https: / / www. feandalucia. ccoo. es/ docu/ p5sd6409.pdf

Requena-Pérez, C. M., Martín-Cuadrado, A. M., \& Lago-M arín, B. S. (2015). Imagen corporal, autoestima, motivación y rendimiento en practicantes de danza. Revista de Psicologia Del D eporte, 24(1), 37-44.

Rodríguez, C., \& Caño, A. (2012). Autoestima en la adolescencia: análisis y estrategias de intervención. International Journal of Psychology and Psychological Therapy, 12(3), 389-403. Retrieved from http:/ / ww w. ijpsy. com/ volumen 12 / num3/ 337/ autoestima-en-la-adolescencia-anlisis-ES. pdf

Rokka, S., Kouli, O., Bebetsos, E., Goulimaris, D., \& M avridis, G. (2019). Effect of dance aerobic programs on intrinsic motivation and perceived task climate in secondary school students. International Journal of Instruction, 12(1), 641-654. https:/ / doi.org/ 10.29333/ iji.2019.12141a

RuedaVillén, B., \& LópezAragón, C. L. (2013). Música y progrma de danza creativa como herramienta expresión de emociones. Retos: N uevas Perspectivas deE ducación Física, D eportey Recreación, 2041, 141- 148.

Sánchez, I., O rdás, R., \& Lluch, Á. (2011). Iniciación a la danza como agente educativo de la expresión corporal en la educación física actual . Aspectos metodológicos. Retos: NuevasTendencias En Educación Física, Deportey Recreación, 2041(20), 33-36. Retrieved from https: / / dialnet. unirioja. es/ servlet/ articulo?codigo $=3713271$

Silva-Escorcia, I. (2015). Autoestima, adolescencia y pedagogía. Revista Electronica Educare, 19(1), 241-256.

Sánchez, J., \& Ruiz, A. (2015). Relationship between self- esteem and body image in children with obesity. Revista Mexicana deTrastornos Alimentarios, 6(1), 3844. doi: https:// doi.org/10.1016/ j.rmta.2015.05.006

Revuelta, L.; Esnaola, I.; Goñi, A. (2016) Relaciones entre el autoconcepto físico y la actividad físico deportiva adolescente. Revista Internacional de M edicina y Ciencias dela Actividad Física y del D eporte/ International Journal of Medicine and Science of Physical Activity and Sport, vol. 16, núm. 63, septiembre, pp. 561-581 Univer sidad Autónoma de Madrid, España

Shavelson, R. J., Hubner, J. J., And Stanton, J. C. (1976). Self-concept: Validation of construct interpretations. Review of Educational Research, 46, 407-441. DOI: 10.3102/00346543046003407

Taberno, C., Serrano, A., \& M érida, R. (2017). Estudio comparativo de la autoestima en escolares de diferente nivel socioeconómico Carmen. Psicología Educativa, 23, 9-17. doi: https:/ / doi.org/ http:/ / dx. doi. org/ 10.1016/ j. pse2017.02.001

Wagener, T. L., Fedele, D. A., Mignogna, M. R., Hester, C. N., \& Gillaspy, S. R. (2012). Psychological effects of dance-based group exergaming in obese adolescents. Pediatric Obesity, 7(5), 1-7. https:// doi.org/ 10.1111/ j.2047-6310.2012.00065.x

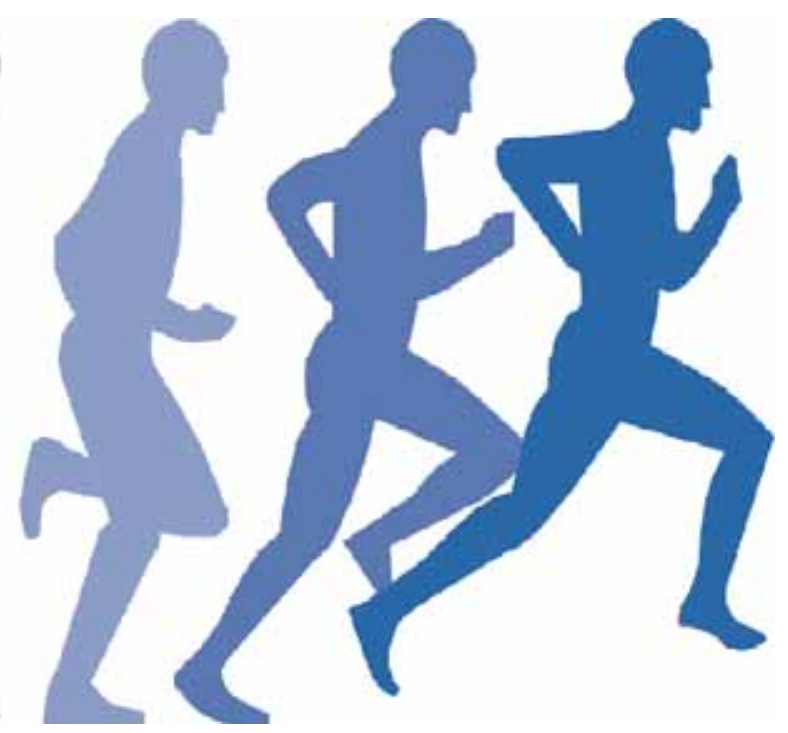

